\title{
La institucionalización de las políticas de igualdad de género en la Universidad española. Avances y retos
}

\author{
Inma PASTOR GOSALBEZ \\ Ana ACOSTA SARMIENTO \\ Universitat Rovira i Virgili \\ inma.pastor@urv.cat \\ ana.acosta@urv.cat
}

Recibido: Junio 2016

Aceptado: Diciembre 2016

\section{RESUMEN}

El camino hacia la eliminación de las brechas de género se mueve entre avances y promesas. Su ritmo va marcado por la combinación de las políticas, la legislación y los cambios por parte de la sociedad. A pesar de las acciones desarrolladas, la desigualdad de género persiste en los campos científicos, tecnológicos y académicos. Los Planes de Igualdad, de obligada implementación en las Universidades españolas desde la entrada en vigor de la Ley Orgánica 3/2007 de 22 de marzo para la igualdad efectiva de mujeres y hombres, constituyen una vía importante, pero no suficiente, para conseguir la igualdad en este ámbito. Un elemento de impulso clave pueden ser las estructuras y redes para la implementación de políticas de igualdad (intra e interuniversitarias, así como con otros organismos e instituciones claves), las cuales no se encuentran suficientemente desarrolladas en la actualidad. En este artículo se expone el proceso de institucionalización de las políticas de igualdad en las universidades acompañado de un análisis de la normativa aplicable al ámbito universitario. Además se presentan datos sobre las unidades de igualdad y sus características y se finaliza con una reflexión sobre los elementos que pueden aumentar el impacto de las estructuras de igualdad propias de las universidades así como de los retos pendientes.

Palabra clave:

Keywords: Género, Universidad, Políticas de igualdad, Unidades de Igualdad, Sociología del género.

\section{The institutionalization of gender equality policies at the Spanish university. Advances and challenges}

\author{
ABSTRACT \\ The path to eliminating gender gaps moves between advances and promises. Its pace is \\ determined by the combination of policies, legislation and social changes. Despite the measures


taken, gender inequality persists in scientific, technological and academic fields. Equality Plans, which must be implemented at Spanish universities since the adoption of the Organic Law 3/2007 of 22 March for the effective equality of women and men, constitute an important, but not sufficient, step to achieve equality in this area. A key factor can be the structures and networks for the implementation of equality policies (intra- and interuniversity ones, as well as those with other agencies and institutions), which are not sufficiently developed at present. This article describes the process of institutionalization of equality policies at Spanish universities and presents an analysis of the legislation applicable to university level. Furthermore, data about equality units and their characteristics are shown. The article concludes with a reflection upon the elements that can increase the impact of university equality structures as well as the remaining challenges.

Keywords: Gender, University, Equality Policies, Equality Units, Sociology of gender.

\section{INTRODUCCIÓN}

Desde el último tercio del siglo XIX comienza la incorporación paulatina de las mujeres a las aulas de la universidad española. Hasta entonces, el acceso a la educación universitaria estaba reservado exclusivamente para los hombres ya que las mujeres estaban relegadas a las esferas domésticas y familiares. Esta apertura de la universidad a las mujeres no sería completa dado que una vez concluían sus estudios, muchas mujeres graduadas encontraban grandes obstáculos en sus esfuerzos para desarrollar las profesiones para las que habían estudiado (Duch, 2014).

Si bien es cierto que, en las últimas décadas, el mercado de trabajo español ha sido testigo de la incorporación masiva de las mujeres, ese dato inicial no puede obviar los matices, barreras y desafíos que nos obliga a continuar señalando que las desigualdades laborales entre hombres y mujeres se han modificado pero no han desaparecido. Estas nuevas fronteras de la desigualdad, como las denominan Maruani, Rogerat y Torns (2011), se hacen evidentes incluso al contemplar un colectivo tan cualificado como el de las académicas.

Si partimos de que una universidad igualitaria es aquella que en la práctica permite que cada uno de los individuos, dependiendo de sus méritos y su esfuerzo, pueda desarrollar su carrera profesional sin interferencias de otro tipo, garantizando la igualdad efectiva de trato y de oportunidades, descubrimos que la universidad no puede considerarse como tal en todos los aspectos. A pesar de que formal y aparentemente todos los miembros de la comunidad universitaria están sometidos a un sistema en el que se miden los méritos, la universidad presenta aún interferencias de tipos diversos, ya sean políticas, de intereses, de prejuicios, siendo estos últimos pero no solo, los que afectan a las mujeres.

Mediante entrevistas a investigadoras principales de grupos de investigación es decir, a mujeres muy bien posicionadas dentro de la academia, Pastor et al. (2015) pudieron comprobar la existencia de sesgos de género dentro de la dinámica universitaria. En esa investigación se constató que existe la percepción de que las mujeres tienen que enfrentarse a una serie de barreras añadidas que no suelen tener los 
varones. Si bien los discursos de las entrevistadas no siempre lo revelaban en un primer momento, a lo largo de las entrevistas en profundidad, éstas mismas investigadoras pasaban de un discurso inicial de negación a una reflexión más profunda y reveladora a medida que avanzaban las entrevistas. Se trata, principalmente, de dos tipos de dificultades, por un lado las derivadas del funcionamiento poco claro y no totalmente meritocrático de la institución y, por otro lado, las dificultades relacionadas con la desigual carga de trabajo reproductivo que asumen hombres y mujeres.

Las medidas de conciliación desarrolladas por las universidades, dentro de las que destacan la flexibilidad horaria, la reducción de la jornada laboral, los permisos retribuidos y licencias sin sueldo (Argüelles, 2011; Gala, 2010) pueden considerarse de gran avance, sin embargo, la vigencia de la división sexual del trabajo en el ámbito universitario continúa siendo uno de los elementos a destacar para explicar las características de la presencia y posición de las mujeres en las mismas (Alcañiz, 2014; Arranz, 2001; León, 2010). Este contexto se caracteriza por tres grandes hechos: por un lado se constata que las mujeres siguen asumiendo gran parte de la carga reproductiva. Si tenemos en cuenta los datos del Instituto Nacional de Estadísticas de España, en su informe Mujeres y hombres en España (2015) "El 91,9\% de las mujeres (de 10 y más años) realizan tareas domésticas y se ocupan del cuidado de niños, ancianos y personas dependientes durante 4 horas y 29 minutos diarios, frente al 74,7\% de los hombres que dedican en promedio 2 horas y 32 minutos" (p. 384). Si bien estos datos son globales de toda la población, los estudios realizados en el ámbito universitario sobre distribución de las cargas del trabajo doméstico muestran que persiste la división sexual también del trabajo doméstico (Pastor, 2008). Además, dicho estudio mostraba no sólo que hombres y mujeres asumen cargas diferentes -en concreto la población estudiada de la URV- sino que su percepción de la carga de trabajo que realmente asumen también es muy diferente: los hombres creen que hacen más de lo que recogen los registros de carga de trabajo.

Por otro lado, se pueden identificar elementos propios de la dinámica universitaria que limita sus condiciones laborales, posibilidades de ascenso y participación en redes estratégicas y de poder. Son diversas las instituciones científicas que han identificado algunos de los obstáculos vinculados a la propia institución que se encuentran las mujeres en su desarrollo profesional. Informes como el del MIT (1999; 2002) apuntan que las mujeres experimentan discriminación en aspectos como la financiación, el espacio, la contratación y la concesión de puestos fijos. Además se produce una marginación de estas mismas investigadoras relacionada con la invisibilidad y la no aceptación como colaboradoras por parte de sus colegas masculinos. Así mismo reciben un menor número de invitaciones que los hombres para participar en actividades profesionales tales como congresos, presentaciones o conferencias, lo que les impide adquirir méritos.

Como dice el propio informe del MIT (1999) la desigualdad de género es tanto una cuestión de realidad como de percepción. Diferentes estudios coinciden en afirmar que las profesoras sienten que son menos apoyadas por sus departamentos y catedráticos que sus colegas masculinos. También apuntan que disponen de menos información relativa a los procesos de promoción y conocen menos los criterios 
aplicables para orientar sus carreras profesionales (Pastor et al., 2008). Por último pueden actuar autolimitaciones tales como las observadas por López-Ibor et al. (2010) en el caso de los estudiantes universitarios. Según este estudio existe una autolimitación de las mujeres a su carrera profesional, detectando -mediante los resultados de su encuesta- que el porcentaje de las estudiantes que tienen un grado de aspiración profesional alto es significativamente inferior al de los estudiantes. La razón de dicha menor aspiración estaría motivada por una anticipación de la necesidad de tener que ralentizar sus carreras profesionales por razones de conciliación.

Estos análisis sitúan en una encrucijada a las instituciones, ya que, como señala el informe ETAN (Comisión Europea, 2001), este menor aprovechamiento del capital femenino representa cuatro amenazas fundamentales: en primer lugar para la equidad, una cuestión de derechos humanos que no se cumple cuando se identifican elementos de discriminación hacia este colectivo. Por otra parte, se ve dañada la excelencia dada la escasa representación y visibilidad que se le da al capital femenino. En la misma línea, se ve afectada también la eficacia, ya que con el envejecimiento poblacional es necesario incorporar a los jóvenes de ambos sexos. Por último, afecta al óptimo rendimiento, ya que renunciar a las capacidades de las mujeres formadas es un gran desperdicio. En la sociedad del conocimiento, donde la generación, el procesamiento y la transmisión de la información se convierten en las fuentes fundamentales de productividad (Castells, 1997) es imperdonable la pérdida de personal altamente cualificado por razones de género.

\section{CARACTERÍSTICAS DE LA DESIGUALDAD POR RAZÓN DE GÉNERO EN LA UNIVERSIDAD EN EL COLECTIVO DOCENTE E INVESTIGADOR}

Desde la década de los años noventa se han llevado a cabo diversos informes y estudios, en diferentes países, sobre la situación de las mujeres en la ciencia. Estudios que han identificado las desigualdades entre hombres y mujeres y han aportado evidencias empíricas de las barreras que existen, para el desarrollo de las carreras profesionales de las mujeres, en el funcionamiento de las instituciones científicas y las universidades. A partir de estos informes se ha venido articulando una serie de acciones para fomentar la igualdad entre hombres y mujeres en la ciencia. Son de destacar los informes sobre la situación de las mujeres en la ciencia (Tabla 1) que han contribuido a visibilizar la situación de las mujeres, ayudando a modelar las políticas al respecto. 
Tabla 1: Informes sobre la situación de las mujeres en la ciencia

\begin{tabular}{|l|l|l|l|}
\hline Título del informe & País & Año & Alcance \\
\hline The Rising Tide & Reino Unido & 1994 & Nacional \\
\hline Excellence in Research & Dinamarca & 1995 & Nacional \\
\hline $\begin{array}{l}\text { Las dimensiones del género en Ciencia y } \\
\text { Tecnología (Capítulo del "Informe Mundial } \\
\text { sobre la Ciencia", UNESCO) }\end{array}$ & & 1996 & Global \\
\hline Women in Academia & Finlandia & 1998 & Nacional \\
\hline $\begin{array}{l}\text { Recommendations for Equal Opportunities for } \\
\text { Women in Science }\end{array}$ & Alemania & 1998 & Nacional \\
\hline $\begin{array}{l}\text { A Study on the Status of Women Faculty in } \\
\text { Science at MIT }\end{array}$ & Estados Unidos & 1999 & Nacional \\
\hline ETAN & Unión Europea & 2000 & Regional \\
\hline $\begin{array}{l}\text { Women in Science and Technology. } \\
\text { The Business Perspective }\end{array}$ & Unión Europea & 2003 & Regional \\
\hline $\begin{array}{l}\text { La situación de las mujeres en el sistema } \\
\text { educativo de ciencia y tecnología en } \\
\text { España y en su contexto internacional }\end{array}$ & España & 2003 & Nacional \\
\hline $\begin{array}{l}\text { She Figures. Statistics and Indicators on } \\
\text { Gender Equality in Science }\end{array}$ & $\begin{array}{l}\text { Comisión } \\
\text { Europea }\end{array}$ & 2009,2012, & Regional \\
\hline $\begin{array}{l}\text { La situación de las mujeres en el Sistema de } \\
\text { Ciencia y Tecnología (FECYT) }\end{array}$ & España & 2004,2007 & Nacional \\
\hline $\begin{array}{l}\text { Igualdad de mujeres y hombres en las } \\
\text { universidades españolas }\end{array}$ & España & 2010 & Nacional \\
\hline $\begin{array}{l}\text { Libro blanco: Situación de las mujeres en la } \\
\text { ciencia española }\end{array}$ & España & 2011 & Nacional \\
\hline Científicas en Cifras & España & 2013 & Nacional \\
\hline $\begin{array}{l}\text { Inclusión del Género en la Investigación y la } \\
\text { Innovación (CSIC) }\end{array}$ & España & 2015 & Nacional \\
\hline
\end{tabular}

Fuente: Elaboración propia 
Los estudios sobre la participación de las mujeres en la universidad y en las instituciones científicas coinciden en la existencia de diferencias sustantivas respecto a sus colegas masculinos. Diferencias que se constatan tanto en la distribución por categorías profesionales como en el acceso a cargos de gestión, dos hechos que podrían tener relación con otras dimensiones de la actividad científica, como la dirección de grupos y proyectos de investigación así como la dirección de tesis doctorales, en las que también se constatan diferencias.

La primera de las desigualdades citadas hace referencia a la menor presencia de mujeres según se avanza hacia categorías académicas superiores. Este hecho, si bien no es específico de la realidad universitaria, ya que se puede identificar en otros espacios laborales, muestra la consistencia de las dificultades que experimentan las mujeres en su desarrollo profesional. Ciñéndonos al ámbito universitario, esta desigualdad queda representada mediante una gráfica que se ha venido a llamar "el diagrama de tijera". Dicha gráfica muestra cómo las mujeres, a pesar de ser mayoría en las aulas (54.9\%), van descendiendo en número a medida que se asciende en la escala universitaria, encontrando, en el curso 2014-2015, solamente un 20.8\% de catedráticas (Fig. 1). (Ministerio de Educación, Cultura y Deporte, 2015)

Fig. 1: Proporción de hombres y mujeres en los diferentes niveles de las universidades públicas españolas. Curso 2014-2015

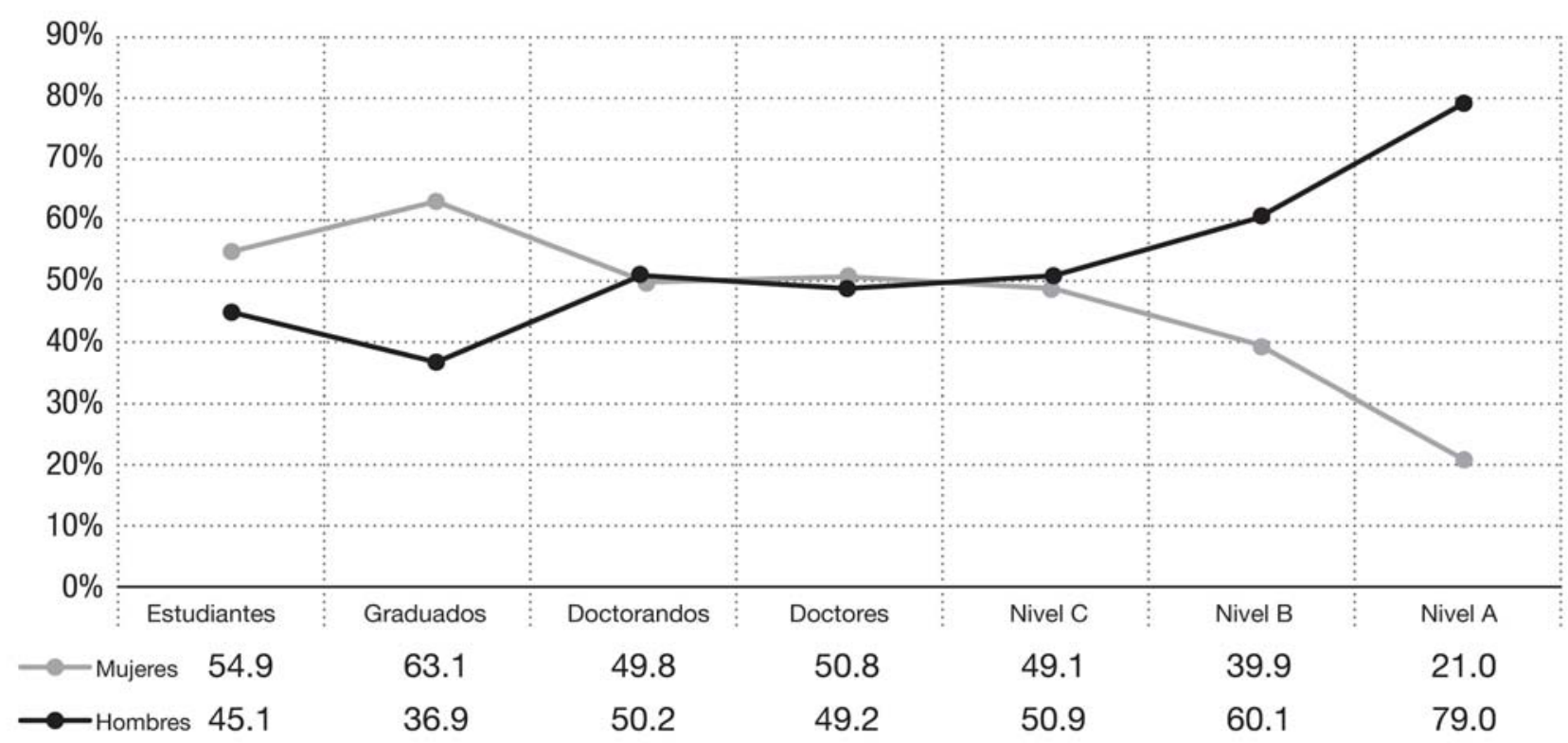

Fuente: Elaboración propia a partir de los datos de Ministerio de Educación (2015)

La agrupación de las categorías se realiza en concordancia con las del informe "Científicas en Cifras" (2013), quedando de la siguiente forma:

- Grado A: Catedráticos/as de Universidad y Profesorado Emérito. 
- Grado B: Catedráticas/os de Escuelas Universitarias, Titulares de Universidad y Profesorado Visitante.

- Grado C: Ayudantes Doctores/as y Contratados/as Doctores/as.

En la gráfica quedan solamente recogidas las mujeres de universidades públicas españolas ya que no ha sido posible acceder a datos equivalentes de las universidades privadas.

Como se aprecia, las mujeres se concentran en puestos con menor prestigio y retribución, alcanzando un porcentaje considerablemente inferior al de los hombres en la cima de la escala académica en el ámbito universitario.

Diversos estudios como FECYT (2004) y Sánchez de Madariaga et al. (2011) coinciden en apuntar que las mujeres no tienen barreras formales para acceder a puestos de personal docente e investigador, es decir para incorporarse como PDI en algunas de las categorías hoy existentes. Los datos muestran que en las categorías iniciales, de entrada a la universidad como espacio profesional, no hay prácticamente diferencias entre hombres y mujeres. Véase por ejemplo los datos correspondientes a la categoría $\mathrm{C}$ de la Figura 1. Sin embargo, las mujeres ven reducida su presencia en los niveles superiores de la carrera académica (véase Niveles A y B de la misma figura 1) así como en los puestos de responsabilidad de la actividad investigadora (por ejemplo como Investigadora principal de proyectos o grupos de investigación (véase figura 2).

Fig. 2: Distribución por sexo del investigador principal de las ayudas del Subprograma Estatal de Generación de Conocimiento (Proyectos de I+D) concedidas a las universidades por áreas de conocimiento (Año 2014)

\begin{tabular}{|c|c|c|}
\hline & Mujeres & Hombres \\
\hline $\begin{array}{l}\text { Humanidades } \\
\text { y Ciencias Sociales }\end{array}$ & $34,5 \%$ & $65,5 \%$ \\
\hline $\begin{array}{l}\text { Tec. de la producción } \\
\text { y las comunicaciones }\end{array}$ & $18,4 \%$ & $81,6 \%$ \\
\hline $\begin{array}{c}\text { Medio ambiente } \\
\text { y recursos naturales }\end{array}$ & $28,6 \%$ & $71,4 \%$ \\
\hline $\begin{array}{l}\text { Ciencias de la vida } \\
\text { y agroalimentación }\end{array}$ & $32,7 \%$ & $67,3 \%$ \\
\hline
\end{tabular}

Fuente: Datos básicos del Sistema universitario español. Curso 2015-2016 (Ministerio de Educación, 2016).

La publicación trienal She Figures. Estadísticas e Indicadores sobre Igualdad de Género en Ciencia, de la CE y el Grupo Helsinki abrió una ventana importante para la recogida de datos sobre la situación de las mujeres en la ciencia, permitiendo así monitorear los avances hacia la igualdad de género en las esferas de la educación 
superior y el mercado laboral en todos los Estados de la UE, y también en su área de influencia (Croacia, Islandia, Noruega, Suiza y Turquía). She figures permite observar la evolución de la situación de las mujeres en la ciencia y comparar las diferencias tanto en el paso del tiempo como las diferentes áreas geográficas.

Los resultados a nivel europeo publicados en She Figures, junto con el análisis de los "libros blancos" en diferentes países, confirman la existencia de diferencias significativas en los procesos de promoción a las cátedras universitarias. También se observó la escasez de mujeres en varios campos científicos. She Figures cuantifica esta segregación y señala la existencia de techo de cristal, barrera invisible pero real que impide a las mujeres llegar a los puestos o nivel más altos, impidiendo sus probabilidades de asumir posiciones de responsabilidad en las universidades. Así, en el ámbito de la educación superior, las investigadoras están más representadas en el campo de las ciencias sociales en 12 de los 28 países, principalmente en los países del sur de Europa y en las más recientes adiciones a la UE. Las mujeres están más representadas en las ciencias médicas en 11 de los 28 países, básicamente, las que incluyen la UE-15 y Japón. En la mayoría de los países (20 de 28), la mayor diferencia se encuentra en el campo de la ingeniería y la menor diferencia se encuentra en las humanidades.

Desde que se inició la publicación de She Figures, los países de la Unión Europea han podido realizar un seguimiento sistemático de los datos sobre la situación de las mujeres científicas. España, además de aportar datos a este informe europeo, es uno de los países que han comenzado a examinar el sesgo de género a través de estudios más exhaustivos como es el caso del Libro Blanco: Situación de las mujeres en la ciencia española (Sánchez de Madariaga y otros, 2011). Uno de los elementos constatados es la diferencia significativa en los procesos de promoción a la cátedra. Los hallazgos corroboran la tendencia reportada por Wennerås y Wold en 1997: la probabilidad de que un profesor titular se convierta en catedrático es 2,5 veces mayor para un hombre que para una mujer, incluso si tienen características similares (la misma edad, el mismo tiempo transcurrido desde el doctorado, el mismo conocimiento disciplinario y la productividad científica). Además, otro factor identificado que afecta negativamente a las mujeres, pero no a los hombres, es la presencia de hijos: un hombre con hijos tiene cuatro veces más probabilidades de ser promovido a profesor titular, que una mujer con hijos aunque sus características profesionales sean similares (Sánchez de Madariaga, 2012).

Por su parte, Zinovyeva y Bagués (2010) han mostrado la existencia de sesgos de género en los procesos de promoción, según el sexo de los miembros del panel de evaluación y la posición a la que los profesores estaban aplicando. En este caso, por cada miembro hombre del panel de evaluación para cátedras, disminuye el número de mujeres seleccionadas en un 14\% (Zinovyeva y Bagués, 2011). En la misma línea Sánchez de Madariaga (2011: 17) afirma que "un evaluador adicional en el comité de siete personas disminuye la probabilidad de promoción de la mujer con respecto al candidato varón en un 14\%".

De esta manera, en línea con lo que sostiene Wenneras y Wold (1997), se puede decir que el menor éxito profesional por parte de las mujeres en la universidad no 
puede ser explicado por falta de productividad científica o académica. Por el contrario, según los estudios de las investigadoras citadas, a igualdad de méritos, la categoría que se obtiene es más baja y el tiempo que se tarda en superar un nivel determinado, es siempre superior al de los hombres. En concreto, según Paloma Alcalá y para el caso español, cuando las mujeres llegan al escalafón más alto han tardado una media de 16 a 20 años más que los varones, citado por Sedeño (2000).

En el caso de la Universitat Rovira i Virgili, antes de diseñar cada uno de los planes de igualdad aprobados, se llevó a cabo, como en casi todas las universidades españolas, un diagnóstico ${ }^{1}$ de la desigualdad de género. La investigación se desarrolló con un enfoque metodológico que combinaba análisis de datos primarios con un estudio cualitativo consistente en entrevistas en profundidad a una muestra de miembros del personal docente e investigador. Los datos permiten dibujar una imagen con respecto a la situación de las mujeres y de los hombres en esta universidad, así como la identificación de las principales desigualdades por razón de género y la recogida de los puntos de vista de los actores involucrados. El análisis de los datos muestra una realidad similar a la de otras universidades si bien, en algunas características, las cifras son levemente diferentes. En su conjunto, la mayoría de los diagnósticos y estudios relativos a las universidades españolas (como recogen Elizondo, Novo \& Silvestre; 2010) coinciden con los informes tanto internacionales (MIT, 1999) como nacionales (Sánchez de Madariaga, 2011), más relevantes al respecto, a la hora de identificar los elementos que caracterizan la desigualdad por razón de género.

En línea con estos diagnósticos y estudios se puede apuntar que hay diferencias entre hombres y mujeres PDI de las universidades en cuanto al grado de titulación, edad, año de contratación y ámbito de conocimiento (variables que se pueden agrupar bajo el nombre de variables de origen). Ante esta realidad surge la siguiente pregunta ¿Podrían estas diferencias ser una explicación del acceso desigual de hombres y mujeres a las categorías laborales de más éxito Siguiendo las conclusiones de Pastor, Valls, Solsona (2010) no de forma totalmente satisfactoria. Es decir, las variables de origen no cuentan toda la desigualdad existente entre hombres y mujeres sino que las trayectorias de hombres y mujeres están marcadas, además, por factores internos al sistema universitario, factores que facilitan la dirección ascendente de la carrera académica de los hombres y ponen en mayor dificultad la de las mujeres.

Las leyes aprobadas en los últimos años constituyen un logro considerable para las mujeres y han permitido avanzar en lo que denominamos institucionalización de las políticas de igualdad. No obstante, coincidimos con Evangelina García Prince (2006) en identificar que ciertos avances han generado un espejismo de igualdad, de manera que se considera la igualdad entre hombres y mujeres un hecho ya alcanzado. Marcela Lagarde (1993) también se ha referido a esta situación con la expresión "velo de la igualdad" para identificar el mecanismo ideológico que oculta o impide ver, en este

\footnotetext{
${ }^{1}$ Según el "Resumen de contenidos" del IX Encuentro de Unidades de Igualdad de las Universidades Españolas celebrado en Madrid los días 12 y 13 de mayo de 2016. Documento no publicado pero disponible entre las Unidades de Igualdad.
} 
caso, la realidad desigual.

En este contexto, la discriminación directa ha dado paso, gradualmente, a una más difícil para detectar, la discriminación indirecta. Tal es el caso del experimento realizado por Moss-Racusin et al. (2012), que demostró que había sesgos de género hacia las mujeres en los departamentos de ciencias, los cuales favorecen a los estudiantes varones. Para validar su hipótesis, los investigadores enviaron exactamente el mismo curriculum, cambiando únicamente los nombres (John o Jennifer) para aplicar a una plaza. Las evaluaciones fueron diferentes según el sexo del solicitante a pesar de tener los mismos méritos. En el caso de John, este fue valorado de manera más positiva, proponiendo además un mayor plan de mentoring y un salario más alto de partida.

Por otro lado, un análisis de la distribución de los premios científicos, los cuales aportan visibilidad, prestigio y recursos económicos a los investigadores, también muestra la existencia de un marcado sesgo de género, tal como lo demuestran Díaz y Gómez (2015). Estos premios, además de aportar reconocimiento, actúan como facilitadores para el impulso de la carrera profesional. A pesar de las dificultades que encontraron las investigadoras para acceder a los datos de postulaciones desagregados por sexo, llegaron a reveladoras conclusiones acerca de la infrarrepresentación de mujeres candidatas a los premios "en aquellos procedimientos que se basan en nominaciones indirectas por parte de la comunidad científica o profesionales de prestigio en otras áreas", contrastando con los premios de postulación directa donde se encontró paridad entre los candidatos. Los premios de postulación indirecta son los más comunes en convocatorias para niveles avanzados de la carrera, mientras que los directos suelen ser dirigidos a investigadores noveles. Asimismo, apuntan las investigadoras "se observa una relación directa entre mayor objetividad y transparencia en requisitos y criterios, y una mayor presencia de mujeres en los galardones" (Díaz y Gómez, 2015: 28)

Por todo lo que hemos visto, el hecho de que la Universidad sea una institución que garantiza la igualdad formal por razón de género no garantiza en la práctica una igualdad real de oportunidades. Paradójicamente, además, esta igualdad formal hace que las desigualdades de género estén marcadas por la invisibilidad y la inconsciencia, aspecto que puede hacer devaluar la percepción de esta desigualdad y la necesidad de aplicar políticas a favor del equilibrio de géneros. Mantenemos que se hace necesario detectar las barreras invisibles objetivas o subjetivas que se encierran en las culturas de las organizaciones, también de las universidades.

\section{PERSPECTIVA DE GÉNERO EN LAS POLÍTICAS EUROPEAS DE CIENCIA Y TECNOLOGÍA}

La situación de las mujeres en la ciencia fue tratada por primera vez en Europa en la década de 1980 en los países nórdicos y Alemania, que publican el primer informe nacional sobre la promoción de las mujeres en el mundo científico en 1989. Este esfuerzo puede ser considerado como el puntapié inicial en el análisis de la igualdad de género en la ciencia y la tecnología. Tales iniciativas se han extendido en la Unión 
Europea, como muestran los documentos publicados por diferentes países, y coinciden en la importancia de desarrollar políticas gubernamentales dirigidas a mejorar la calidad de la ciencia a través del empoderamiento de las comunidades científicas y particularmente del papel de la mujer en las mismas.

Presentar el tema de la incorporación de la perspectiva de género en la conferencia de la Mujer y Ciencia en 1998 se considera otro punto de inflexión importante para la comunidad científica de la UE ya que un año más tarde, la Comisión Europea (CE) presentó la comunicación "Mujeres y ciencia: movilizar a las mujeres para enriquecer la investigación europea", de 17 de febrero de 1999 proponiendo una estrategia coherente dentro del Quinto Programa Marco (1998-2002) con el fin de promover la investigación sobre y de mujeres. En esta ocasión se hacía hincapié en la necesidad de mantener una discusión dinámica sobre la situación de las mujeres en la ciencia, el desarrollo de mejores indicadores de desigualdad y la necesidad de compartir experiencias sobre estos temas. Estas prioridades se establecieron siguiendo criterios comunes para los Estados miembros, el Parlamento Europeo y la Comisión Europea.

En el año 2001 la Comisión Europea publicó el informe de la Red Europea de Evaluación de Tecnología (ETAN) sobre la promoción de la excelencia a través de la integración de la igualdad de género, con la intención de mejorar la situación de las mujeres en la ciencia y el desarrollo de las políticas científicas. En concreto, el informe fue diseñado para influir en las políticas de dos maneras: a) la integración de la igualdad de género en las diferentes instituciones de la enseñanza y la ciencia a través de la financiación, la formación y contratación de personal científico, b) el logro de la igualdad en las estructuras científicas y académicas, en la revisión por pares, la financiación, políticas de liderazgo y ajuste de las prioridades académicas.

Esa iniciativa de transversalidad de género se ha continuado implementando en los programas marco europeo (VI y VII). Un ejemplo de esto es que la CE estableció el objetivo de apoyar las acciones que buscan lograr un cambio estructural y cultural en la forma en que el género y la diversidad se gestionan en las universidades y centros de investigación. Su objetivo es conseguir que las universidades y centros de investigación cooperen en las acciones conjuntas de desarrollo de estrategias para aumentar la participación de las mujeres y el impulso de sus carreras. Universidades y centros de investigación han sido animados a intercambiar las mejores prácticas y crear planes de acción para lograr el cambio estructural y para hacer un mejor uso de la diversidad. De este modo, la UE reconoce que la integración de la dimensión de género en el diseño, evaluación y ejecución de la investigación es todavía muy limitada. En consecuencia, las universidades se enfrentan al reto de mejorar todos aquellos aspectos con el fin de mejorar la calidad y pertinencia de la investigación.

Estas iniciativas han tenido continuación en el programa H2020 y se han materializado, especialmente, en el impulso a la introducción de la dimensión de género en los contenidos de la investigación y la innovación. En concreto, dos estrategias se han desarrollado dentro del nuevo programa marco: por un lado, corregir la desigualdad en la participación en la investigación en todas las etapas de la carrera científica y en diferentes campos de la investigación, para ello la $\mathrm{CE}$ ha declarado su 
compromiso de dedicar el $40 \%$ de miembros de todos los expertos grupos, juntas y comités al sexo menos representado; por otro lado, considerar el género como una dimensión de investigación teniendo en cuenta las diferencias biológicas, sociales y económicas entre hombres y mujeres en todos los procesos de investigación. De hecho, todos los programas incluidos en H2020 deben contener información sobre cómo hacer frente a la desigualdad de género y la forma de integrar la dimensión de género. Esto también debe reflejarse en nuevos proyectos, exigiendo el compromiso de las instituciones de investigación incluyéndolo en las cláusulas del acuerdo. Además, hay cuatro programas específicos de investigación y de apoyo a las estructuras de género. Por lo tanto, H2020 representa un paso adelante en la estrategia europea sobre la incorporación de la dimensión de género.

Además de la estrategia en materia de políticas científicas, la sensibilización hacia la necesidad de tratamiento de género y la igualdad de oportunidades, se refleja en las acciones legales de la UE entre 2000-2010, como Directiva 2002/73 / CE del Parlamento Europeo y del Consejo relativa a la aplicación de la igualdad de trato entre hombres y mujeres en el acceso al empleo, la formación, las condiciones de promoción y de trabajo profesional; y la Directiva 2004/113 / CE del Consejo que aplica el principio de igualdad de trato de ambos sexos con respecto al acceso a bienes y servicios y su suministro.

Por otra parte, en 2006 la Comisión Europea, en el Reglamento (CE) $n^{\circ}$ 1922/2006, crea el Instituto Europeo de la Igualdad de Género (EIGE) como instrumento para difundir información, intercambiar buenas prácticas y desarrollar nuevos instrumentos metodológicos que promuevan la incorporación de la perspectiva de género (integración de la dimensión de género en todas las políticas), asignándole los siguientes objetivos generales (art. 2): «Contribuir a la promoción de la igualdad de género y reforzarla, incluida la incorporación de la perspectiva de género a todas las políticas comunitarias y en las políticas nacionales resultantes, luchar contra la discriminación por motivos de sexo y dar a conocer mejor las cuestiones relacionadas con la igualdad de género a los ciudadanos de la UE con la prestación de asistencia técnica a las instituciones comunitarias, en particular a la Comisión, y a las autoridades de los Estados miembros, tal como se indica en el artículo 3 del Reglamento por el que se crea un Instituto Europeo de la Igualdad de Género». El EIGE obtuvo su independencia financiera y administrativa en junio de 2010, año en el que se aprobó la estrategia para la igualdad de género 2010-2015, elaborada por la CE y adoptada por el EIGE. (Instituto Europeo de la Igualdad de Género, 2011).

\section{AVANCES EN LA UNIVERSIDAD ESPAÑOLA Y LA IGUALDAD DE GÉNERO}

\subsection{LEGISLACIÓN}

Además de la normativa europea, cada país tiene una legislación específica en 
materia de igualdad de género. En el caso de la legislación de España, se expresan claramente los principios de igualdad específicos en el artículo 14 de la Constitución Española que establece que todos los españoles son iguales y no pueden ser objeto de discriminación por razón de sexo. También en el artículo 9.2 que exige que los poderes públicos deben promover las condiciones que garanticen la igualdad de los individuos así como en el artículo 35 que garantiza a los españoles el derecho a la promoción en el trabajo y prohíbe la discriminación laboral por razón de sexo.

En 2007, España aprobó la Ley Orgánica 3/2007 (comúnmente conocida como la Ley de Igualdad), que tiene el doble propósito de avanzar en la lucha contra la discriminación de género y dar prioridad a la promoción de la igualdad de género desde una perspectiva interdisciplinaria en diversos ámbitos.

En virtud de la Ley de Igualdad, las autoridades públicas deben asegurar la eficacia de las medidas de transformación destinadas a garantizar la igualdad de trato y de oportunidades, y la administración pública debe evaluar periódicamente sus políticas. La Ley de Igualdad exige la elaboración de planes de igualdad como medida para promover la igualdad, así como la visibilidad de las estadísticas desagregadas. También obliga a la administración a promover la educación y la investigación en materia de igualdad, ofreciendo títulos de postgrado y de apoyo a la investigación en esta área. Las universidades, como instituciones de la administración pública, están obligadas a desarrollarlos.

En concreto, los artículos 23 a 25 de la Ley de Igualdad contienen disposiciones relativas a la igualdad de género en la educación. El artículo 25 , se refiere a la educación y la investigación en materia de igualdad de género, así como la promoción de medidas concretas: la inclusión de temas de igualdad en los programas de educación superior, la creación de programas de postgrado en materia de igualdad específicos, y la promoción de investigación especializados en el ámbito de la igualdad. La Ley de Igualdad no hace referencia al principio de la representación equilibrada en los órganos universitarios. Esta omisión es notable, teniendo en cuenta que las mujeres están subrepresentadas en los órganos de decisión de la universidad en España (Nieves, 2008).

Por su parte, la Ley Orgánica 4/2007, de Universidades (Boletín Oficial del Estado (BOE) no. 89, abril, 13 de 2007) muestra, en sus artículos, una sensibilidad especial hacia la igualdad de género que faltaba en la Ley Orgánica 6/2001 de Universidades (diciembre de 2001). La nueva Ley demanda un mayor compromiso con la igualdad de género y rellena los huecos dejados por la Ley de Igualdad. En el preámbulo de esta ley destaca: "el papel de las universidades como transmisores fundamentales de los valores", y agrega: "El desafío que enfrenta la sociedad actual para convertirse en una sociedad tolerante e igualitaria que respete los derechos y libertades fundamentales y de la igualdad entre hombres y mujeres- deben, sin duda, extenderse a las universidades". La Ley Orgánica 4/2007 exige una serie de medidas encaminadas a lograr la paridad en los órganos de representación y de gobierno de las universidades, así como una mayor participación de las mujeres en los grupos de investigación y en las altas esferas de la universidad profesores y personal de investigación "acorde con el porcentaje de graduados universitarios que son mujeres". 
La ley también hace un llamado a las universidades para crear unidades de igualdad de género "para llevar a cabo las funciones relacionadas con el principio de igualdad entre mujeres y hombres" (Disposición Adicional 12). Este último elemento, la creación de las unidades de igualdad, supone un avance más en el proceso de institucionalización de las políticas de igualdad en el ámbito universitario. Este proceso se aceleró a partir de la Ley Orgánica de 3/2007, aunque desde la Ley Orgánica 1/2004 ya se habían comenzado a impulsar en las universidades la necesidad de abordar una formación que garantizara el respeto y la promoción de la igualdad de género, especialmente en lo que respecta a la lucha machista. También cabe destacar la inclusión en la, comúnmente llamada, Ley de la Ciencia, la Ley 14/2011, de 1 de junio, de la Ciencia, la Tecnología y la Innovación la disposición adicional decimotercera dedicada a detallar los mecanismos que prevé la Ley para materializar la implantación de la perspectiva de género. De esta disposición se puede destacar la voluntad de incorporar la perspectiva de género como una categoría transversal en todos los aspectos de la actividad científica así como el empuje a la composición paritaria de comités y comisiones. Así también se recoge la obligación, para los Organismos Públicos de Investigación, de adoptar Planes de Igualdad y de "incluir medidas incentivadoras para aquellos centros que mejoren los indicadores de género en el correspondiente seguimiento anual".

Se puede afirmar, de esta manera, que, como fruto del desarrollo legislativo, pero también -y tal vez como verdadero motor- a partir de la demanda de grupos de investigación en estudios de mujeres y género, las universidades españolas han ido institucionalizando el desarrollo de políticas de igualdad. Este desarrollo se ha producido de forma bastante similar en todas ellas si bien se dan diferencias tanto en el ritmo como en la intensidad de las medidas. El primer mecanismo desarrollado, y que nos permite reconocer la institucionalización de dichas políticas, ha sido la creación de estructuras dentro del organigrama universitario, dedicadas al impulso de planes y políticas de igualdad, las llamadas unidades, oficinas, centros, áreas u observatorios de igualdad $^{2}$. De esta forma, en los últimos años, un elevado porcentaje de las universidades españolas cuentan con un área dedicada a la igualdad, así como un Plan estratégico para conseguirla, encontrándose algunas en su segunda o tercera edición. En el apartado siguiente se presentan algunos datos que describen la situación de las universidades españolas en relación a este punto.

Sin embargo, esto no garantiza que realmente se den pasos firmes hacia la igualdad de género efectiva tal y como muestran los datos presentados en el apartado 2 que ilustra la persistencia de dificultades en el desarrollo profesional de las académicas. Y tras esta contradicción se encuentra un interrogante que necesita ser contestado y a cuya respuesta este artículo quiere contribuir. ¿Cómo puede ser que a pesar del tiempo que se lleva trabajando en políticas de igualdad en las universidades, los cambios sean de tan baja intensidad? El primer paso para contestar esta pregunta es identificar los factores que pueden estar detrás de la baja intensidad de los cambios a favor de la igualdad de género. El bajo compromiso institucional, el presupuesto que

\footnotetext{
${ }^{2}$ Esta definición es adoptada por cada universidad en el momento de creación de las mismas. Su definición y funciones suelen quedar aprobada en Consejo de Gobierno, y en menor medida queda plasmada en los Estatutos de la Universidad.
} 
no pasa de lo simbólico, o la falta de bagaje "académico en el ámbito de estudios de género" de algunas de las responsables, entre otros factores que más adelante analizaremos, podrían explicar que la igualdad se mantenga como una asignatura pendiente en el campo universitario.

\subsection{LAS ESTRUCTURAS PARA EL IMPULSO DE LA IGUALDAD EN LAS UNIVERSIDADES ESPAÑOLAS}

Como se acaba de apuntar, la realidad de las universidades españolas en relación al desarrollo de las políticas de igualdad, es diverso y dispar si bien se dan algunos elementos de similitud. La similitud viene condicionada por el mandato legal de tener una estructura dentro del organigrama que impulse un plan de igualdad así como la existencia de redes formales e informales que orientan el sentido de las políticas de igualdad por razón de género.

La diversidad de las universidades españolas puede ser explicada en base a múltiples y diversos factores; como el tamaño, las fuentes de financiación, la antigüedad y las características sociolaborales. Estas variables junto con otras como la orientación académica de las mismas (es decir si son universidades de orientación general o técnicas) o el rol de los gobiernos autonómicos, pueden ayudar a explicar la heterogeneidad entre las universidades. Sin embargo para un análisis específico de los factores que pueden incidir en una mayor o menor apuesta por las políticas de igualdad, entendemos que es necesario considerar otras características directamente relacionadas con la igualdad. A continuación se presenta información relativa a aquellas variables que se han tenido en cuenta como potenciales factores explicativos de la diversidad entre universidades en materia de políticas de igualdad.

Las variables consideradas, así como la información relativa a las mismas se presentan a continuación en forma de figuras. La existencia de grupos de estudios de género, la participación en redes de igualdad (Figura 3), el lugar de la organización desde dónde son promovidas las políticas universitarias, el tipo de estructura de promoción de la igualdad (Figura 4), el año de creación de la estructura de igualdad (Figura 5), el estado en el que se encuentra el plan de igualdad (elaboración, I Plan, II Plan o III Plan) (Figura 6), el perfil de la responsable de la estructura de promoción de igualdad, la red de contactos, son, entre otros los factores que pueden influir en el diseño e implementación de estas políticas. A continuación se presentan algunos datos que permiten conocer el estado de la cuestión en las universidades españolas. 
Fig. 3: Pertenencia a la Red de Unidades de Igualdad de Género para la Excelencia Universitaria (RUIGEU)

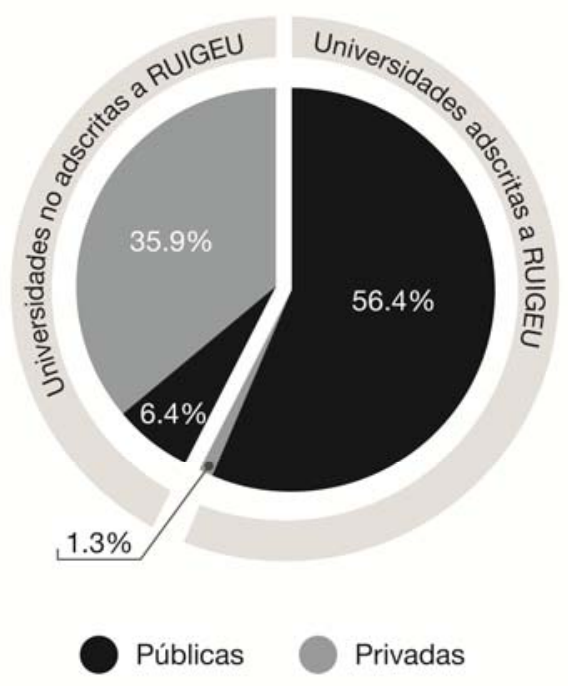

Fig. 5: Año de creación de la estructura de promoción de la igualdad

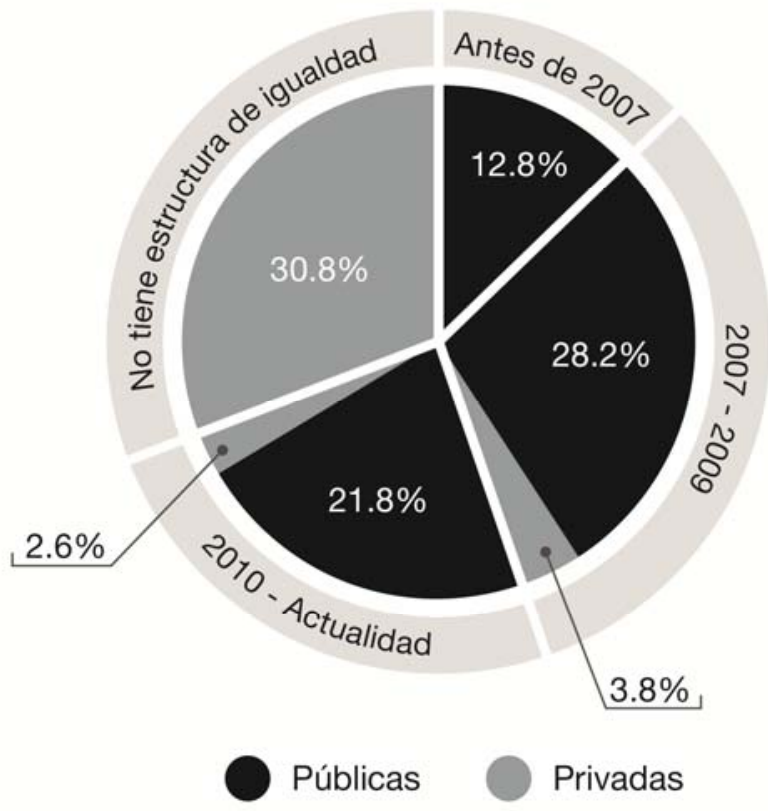

Fuente: Elaboración propia
Fig. 4: Tipo de estructura de promoción de igualdad de las universidades españolas

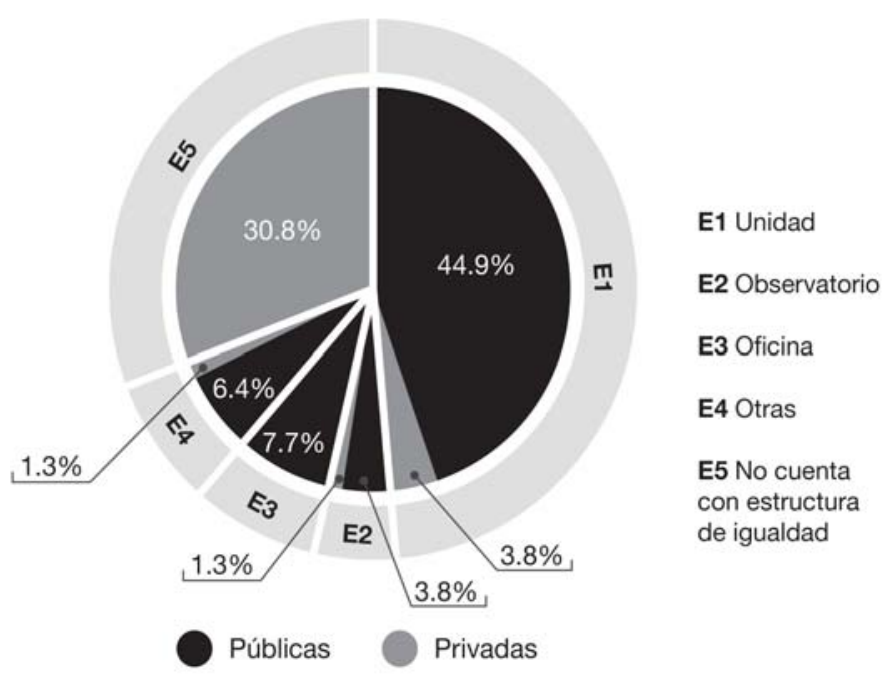

Fig. 6: Estado o fase del plan de igualdad

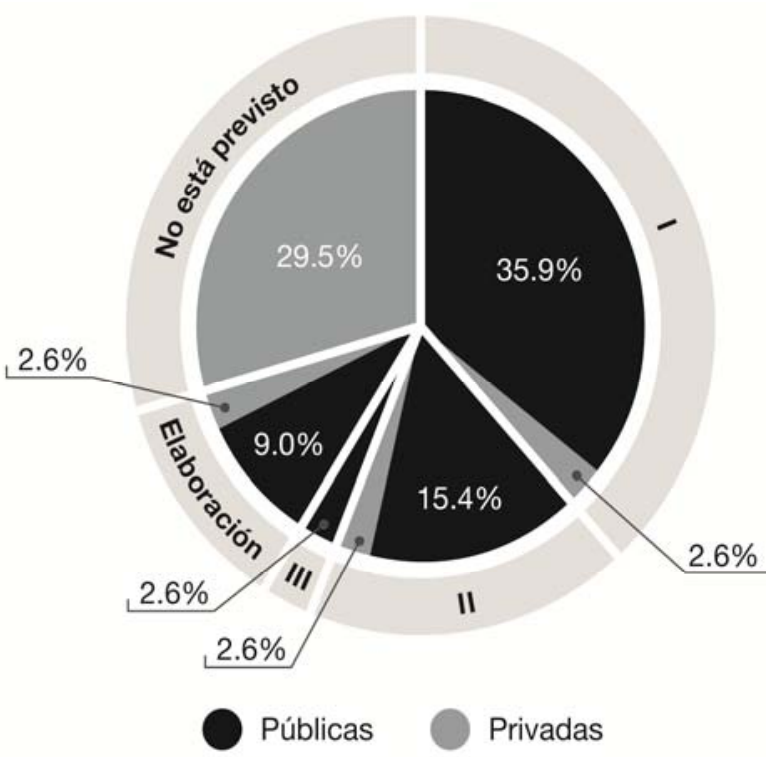


Analizando la cronología de la creación de las estructuras universitarias de promoción de la igualdad en las diversas universidades públicas podemos apreciar un universo complejo y nada uniforme, encontrando que el $21 \%$ de las universidades contaban con área de igualdad anterior incluso a la Ley 3/2007. El mayor porcentaje de universidades $(44.9 \%)$ creó su estructura de promoción de igualdad justamente después de la aprobación de la ley, incluyendo en este grupo a las universidades que crearon unidades los años 2007, 2008 y 2009. A partir del año 2010 se han ido sumando el resto de las universidades para cumplir este mandato legislativo; universidades que representan casi el $45 \%$ del total. En el caso de las universidades privadas, solamente 5 cuentan con estructuras de igualdad, quedando 24 universidades sin ninguna unidad que se haga cargo de este tipo de políticas.

Existen factores importantes que pueden condicionar el inicio del trabajo en políticas de igualdad de las universidades: el interno, con la presión desde las activistas de género y/o una coyuntura política favorable al diálogo por parte del equipo rectoral, y la presión externa de institucionalizar las políticas de igualdad en toda la universidad que es una derivada de todo el trabajo de la Unión Europea y los avances legislativos.

Como decíamos antes, puede ser relevante el papel jugado por las instituciones políticas autonómicas. En algunos casos, como el de Cataluña, hay que apuntar la existencia de un catalizador importante de origen externo a cada una de las universidades pero interno al propio sistema universitario en su conjunto. Nos referimos a la creación, para toda Cataluña, dentro del Consejo Interuniversitario en el que están representados los Rectores de todas las universidades catalanas, de la Comisión Mujer y Ciencia, en el marco de la cual se impulsó que todas las universidades incorporasen de manera institucional una línea de igualdad, desarrollando todas las acciones necesarias para conseguirla. En el conjunto de España no se ha conseguido que el tema de género tenga protagonismo en la Conferencia de Rectores de la Universidad Española (CRUE). Esta sigue siendo una petición reiterada por las representantes de las Unidades de Igualdad en los diversos encuentros que se han realizado desde 2008 -año de realización del primer encuentro ${ }^{3}$ - y que se han consolidado como punto de debate y articulación de propuestas que impulsen las políticas de género en las universidades.

A pesar de la falta de homogeneidad formal y conceptual, las estrategias y líneas de acción que se exponen los planes de igualdad, están articulados en siete categorías que ilustran las principales líneas de trabajo llevado a cabo por las universidades españolas en el ámbito de la igualdad de género:

- Garantizar el principio de igualdad de oportunidades y de trato.

- Promover la compatibilidad de trabajo / familia y la corresponsabilidad.

- Asegurar la participación y representación equilibrada.

- Sensibilizar y aumentar la visibilidad de las mujeres.

\footnotetext{
${ }^{3}$ En el último encuentro, realizado en la UAM en mayo de 2016, se volvió a plantear la necesidad de que este tema cuente con impulso y apoyo desde la CRUE.
} 
- Incorporar la perspectiva de género en la enseñanza y la investigación.

- Incorporar el mainstreaming de género.

- Promover la salud y seguridad en el trabajo.

Mención especial cabe hacer respecto a la lucha contra la Violencia de género ya que, si bien, no constituye una línea de trabajo explícita en el conjunto de los planes de igualdad, existe un volumen importante de unidades de igualdad que están desarrollando acciones específicas. Además, existe un consenso político sobre la importancia de implicar a las universidades en esa lucha, como muestra el hecho de que en todos los encuentros estatales de unidades de igualdad se ha venido tratando el tema.

Analizando su dependencia funcional y política, remitiéndonos a la clasificación descrita por Cerdá (2010), las unidades de igualdad quedan configuradas como "el órgano técnico de referencia en la respectiva comunidad universitaria", definición que le aporta a las universidades un amplio margen para la creación de sus propias estructuras acordes a la visión de cada universidad, tal y como se observa en la figura 7.

Fig. 7: Dependencia de la unidad de igualdad

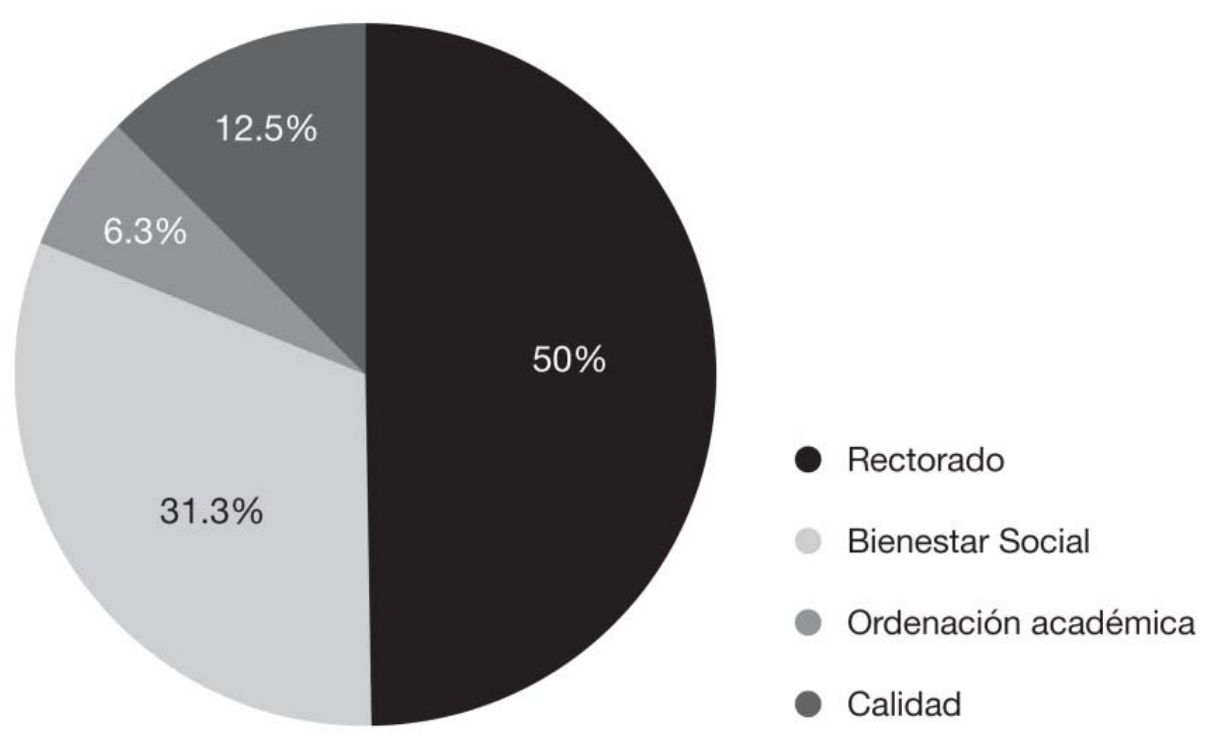

Fuente: Elaboración propia a partir de Cerdá (2010)

De este modo, se pueden identificar cuatro modelos: dependiente del Rectorado (con o sin representación en Consejo de Dirección) o perteneciente a las áreas de Bienestar Social, de Ordenación Académica o de Calidad. 


\section{ELEMENTOS PARA HACER AVANZAR LAS POLÍTICAS DE IGUALDAD EN LAS UNIVERSIDADES}

A nuestro juicio, este proceso -previamente expuesto- de institucionalización de las políticas de igualdad en las universidades, es muy valioso ya que permite avanzar en la eliminación de ciertas discriminaciones que han afectado y todavía afectan negativamente a las mujeres. Entendemos que dos factores, dentro de todo este proceso, destacan por la potencialidad que tienen en contribuir a construir universidades más igualitarias: la creación de unidades de igualdad dentro de la estructura universitaria y la elaboración de planes de igualdad. Esta potencialidad requiere, sin embargo, de algunos elementos clave para el desarrollo de una política de igualdad productiva y coherente, como los que señalan Pastor, Pontón, Belzunegui, Acosta (2015). Para que se consoliden las políticas de igualdad en las universidades es importante la institucionalización citada pero a su vez este proceso debe poner la prioridad en la efectiva mejora de la realidad de las mujeres y no sólo en el mero cumplimiento de las leyes. Cabe apuntar también la existencia de diversos factores derivados de las condiciones actuales de producción del conocimiento que estarían dificultando, en estos momentos, la efectividad de las políticas de igualdad. A nuestro entender, y a modo de hipótesis por contrastar, estos serían por ejemplo: el incremento de competitividad, entre los trabajadores de la ciencia -ya sean profesores de las diferentes categorías o personal investigador en general; la lógica cuantificadora de la actividad científica que no reconoce con el mismo valor a todas las tareas que son requeridas en dicha actividad; un contexto de austeridad que reduce los recursos a invertir en ciencia y tecnología. Esta línea de análisis se ha desarrollado como análisis crítico de las actuales condiciones de trabajo en las universidades y su impacto en la producción del conocimiento (Cuninghame, 2015). Sin embargo queda por desarrollar el análisis necesario sobre el impacto de esas mismas condiciones en el desarrollo de las políticas de igualdad.

Entendemos que la efectividad de las políticas de igualdad en la universidad pasa por incorporar, al trabajo diario de las universidades $y$, en concreto de las unidades $u$ observatorios de igualdad, elementos clave para conseguir una transformación de la academia y el funcionamiento de las instituciones científicas en una dirección que supere las desigualdades basadas en el género. Algunos de estos elementos son los que a continuación se exponen:

- El análisis y la visibilización de datos desagregados por sexo: Este elemento tiene una importancia clave ya que un diagnóstico minucioso y basado en las más estrictas líneas académicas favorece la aceptación por parte de la universidad, atendiendo a que el conocimiento es el elemento articulador. Dar forma de datos, de artículos, de informes, de diagnóstico, a la discriminación por razón de género, es imprescindible para favorecer el debate por parte de la comunidad universitaria. En este sentido, todas las acciones que impliquen diagnosticar y dar a conocer ese diagnóstico son necesarias. Este es un punto esencial en esta primera batería de acciones.

- La promoción y consolidación del trabajo conjunto de las unidades de igualdad con 
la red de expertas en materia de género en cada Universidad: Entendemos necesaria la colaboración de las estructuras universitarias que deben impulsar las políticas de igualdad, con las académicas especialistas en estudios de género. Esta colaboración se nos presenta como imprescindible tanto para identificar las nuevas desigualdades de género como para trasvasar el conocimiento generado por la investigación tanto a la docencia como a las propias políticas. De hecho, nuestros datos ${ }^{4}$ muestran que la existencia anterior (o no) de los grupos de estudio de género, la trayectoria desarrollada y su tamaño se presentan como factores relevantes que explican las diferencias entre las universidades en cuanto a la rapidez y a la forma que toma el proceso de institucionalización que estamos analizando.

- La incorporación de la perspectiva de género en la docencia y la investigación: Consideramos esencial que una masa crítica de mujeres y hombres puedan incorporar la perspectiva de género en la docencia y la investigación. Para ello se hace necesario articular grupos de investigadores y profesores que ya habían introducido la variable de género en sus programas y su investigación.

- El desarrollo de programas específicos de liderazgo o mentoring para mujeres académicas: La experiencia en algunas universidades, como la Universidad del País Vasco, o comunidades autónomas, como Cataluña, en línea con experiencias internacionales muestran la conveniencia de apoyar las carreras profesionales de las mujeres mediante la figura de la mentora y desarrollo de habilidades específicas de liderazgo.

A su vez, entendemos que el proceso de institucionalización de las políticas de igualdad tiene por delante retos a superar que tienen que ver con las dinámicas de la práctica científica. Los retos más relevantes a nuestro entender serían:

- La falta de discusión entre las ramas del conocimiento o los campos académicos: Una característica de las universidades que pueden hacer que sea difícil para trabajar en el desarrollo de planes y unidades de género es la falta de discusión entre los campos académicos. Puede resultar muy importante incluir esta dinámica para una mayor implicación de la comunidad académica.

- La invisibilidad de algunas formas de discriminación: Como se ha apuntado anteriormente, los estudios realizados muestran que la desigualdad de género en las universidades se presentan bajo el espejismo de la igualdad formal y la creencia en la meritocracia (Arranz, 2001). Esto confiere una dificultad ${ }^{5}$ añadida al trabajo de las unidades de igualdad. La existencia de estas unidades presupone la posibilidad de ser capaz de ver y denunciar los casos en que las mujeres, puedan resultar discriminadas. Por lo tanto, el mayor reto es la detección de los casos

\footnotetext{
${ }^{4}$ Tesis doctoral en elaboración: "Las Políticas de Igualdad en las Universidades Españolas. Un estudio de redes".

5 Además las universidades tienen una forma mayormente descentralizada de gestión y funcionamiento que hace que sea difícil trabajar para difundir un nuevo mensaje a todos los servicios y departamentos.
} 
fundamentales de discriminaciones indirectas, invisibles o informales.

- La mentalidad androcéntrica que aún predomina en la comunidad académica: Los prejuicios sexistas y el machismo aún están presentes y condicionan el desarrollo de políticas de igualdad de género y su aplicación.

La presencia y características de estos procesos puede darnos pistas para orientar a las universidades hacia unas políticas de igualdad profundas y transformadoras más allá de la formalidad.

\section{CONCLUSIONES}

Estas reflexiones nos llevan a algunas conclusiones finales. En primer lugar, la legislación española actual no es suficiente para alcanzar la igualdad real entre mujeres y hombres. Las leyes actuales marcan las medidas necesarias, pero no garantizan que tales medidas en última instancia, serán puestas en práctica y lograr los resultados esperados. Además, teniendo en cuenta las dinámicas y actitudes que aún persisten en las universidades españolas hacia la desigualdad, es razonable suponer que la intervención legal en este tema seguirá siendo necesaria en el futuro inmediato. Al hilo de las evidencias de los diferentes estudios existentes, para evitar las discriminaciones por razón de género, son necesarios cambios que pasan fundamentalmente por modificar la manera de funcionar de la universidad, y que, probablemente, no quedarán recogidos en un plan de igualdad.

Formalmente se han ido dando avances importantes que van en la línea de consolidar la meritocracia. En esta línea cabe destacar los procesos de acreditación externa a las universidades y la publicitación de los requisitos necesarios para ello, así como la exigencia de méritos de investigación para participar de determinados procesos de selección. Esta es una buena noticia especialmente para las mujeres ya que hace posible que se vayan dando pasos para evitar los sesgos de género que se producen. Estos pasos son posibles gracias a que cada vez hay más transparencia en los procesos y más claridad en los criterios que se aplican tanto en el acceso como en la promoción dentro de las universidades en general para todos. En nuestra opinión, uno de los factores que más hará avanzar la situación de las mujeres son la claridad y la transparencia de los criterios y procedimientos de la universidad y especialmente en los procesos tanto de acceso como de promoción. En la medida en la que sean más transparentes y claros, resultará beneficiada toda la institución, y por lo tanto también las mujeres, protegiendo a la organización de la influencia de esos otros criterios habitualmente presentes, si bien de manera oculta.

Desde el feminismo académico y, especialmente, las expertas en estudios de género, además de promover la incorporación de las mujeres y su desarrollo profesional en las instituciones académicas y científicas, se defiende, como vía prioritaria para eliminar la desigualdad por razón de género, la necesidad de transformar el conocimiento. Quitar el sesgo de género o "desgenerar la ciencia" en el sentido que apuntaba Susan Harding (1996) se convierte en una línea de actuación en consonancia con otras iniciativas internacionales, como el programa "Gendered 
Innovations" ${ }^{\prime 6}$ de la Universidad de Stanford que cuenta con el apoyo de Comisión Europea y de la National Science Foundation de Estados Unidos. A nuestro entender, hay que tener en cuenta que las mujeres sólo serán capaces de transformar el conocimiento si están bien posicionadas en los lugares en los que se define el valor de los conocimientos. El aumento del número de mujeres en estos lugares no garantiza el cambio, pero sin duda aumenta las posibilidades de progreso. Coincidimos con Schiebinger (2007) en afirmar que el desarrollo de la perspectiva de género en las investigaciones, así como una mayor participación de las mujeres en las mismas añaden creatividad, nuevas preguntas y un abanico de nuevas áreas de investigación por desarrollar.

A la luz de lo expuesto en este artículo, podemos concluir que, si bien la institucionalización de las políticas de igualdad en las universidades españolas pone las bases para hacer avanzar al conjunto de las universidades en la consecución de la igualdad real, son muchos los retos pendientes. A nuestro entender, sólo si se identifican correctamente los elementos que pueden dificultar el avance, se podrán impulsar las medidas más adecuadas. El trabajo en red de las unidades de igualdad junto con otras instituciones dedicadas a la promoción de la igualdad así como el debate académico y científico junto con los grupos de estudios de género, pueden ser un elemento impulsor que haga avanzar la consecución de la igualdad efectiva entre hombres y mujeres.

\section{REFERENCIAS}

ALCAÑIZ, MERCEDES (2014): La conciliación de la vida laboral y familiar en la Universitat Jaume I. Castellón de la Plana: Publicacions de la Universitat Jaume I.

ARGÜELLES BLANCO, ANA ROSA (2011): Conciliación y corresponsabilidad en la ordenación del tiempo de trabajo: comentario de las disposiciones normativas vigentes en las universidades públicas catalanas. RIDEG: revista interdisciplinar de estudios de género, (1), 0015-29.

ARRANZ, FÁTIMA (2001): Hombres y mujeres en el profesorado: un análisis de género en las académicas. In: M. García de León y M.García de Cortázar (ed.), Profesorado universitario y género, 337-404.

CASTELLS, MANUEL (1997): La era de la información: Economía, Sociedad y Cultura. Vol.1 La Sociedad Red. Madrid: Alianza Editorial

CERDÁ, MARÍA ROSA (2010): Modelos de desarrollo de unidades de igualdad en la universidad pública. In Investigaciones multidisciplinares en género: II Congreso Universitario Nacional" Investigación y Género": Sevilla, 17 y 18 de junio de 2010 (pp. 233-247).

\footnotetext{
${ }^{6} \mathrm{https}: / /$ genderedinnovations.stanford.edu/
} 
COMISIÓN EUROPEA (2001): Informe ETAN. Política científica de la Unión Europea. Promover la excelencia mediante la integración de la igualdad entre géneros. Brusselles: Comisión Europea.

CUNINGHAME, PATRICK (2014): La Doble Crisis de la Universidad: Capitalismo Cognitivo, Precariedad Laboral, Producción del Conocimiento y Conflictos Sociales. Ponencia completa para Tema 8, Sociología de la Educación, del Segundo Encuentro de Sociología en la Universidad Autónoma Metropolitana 2014 "La Sociología Hoy...en México y en la UAM", 20 de octubre de 2014, Club Alemán, México DF

DUCH, MONTSERRAT (2014): A empentes i rodolons. Els obstacles sexistes en l'accés de les dones a la universitat espanyola. In: I. Pastor et al. (ed.),Cent anys de dones a la universitat. Tarragona: Publicacions de la URV.

DÍAZ, CAPITOLINA. Y GÓMEZ, Araceli (2015). Las mujeres en los premios científicos en España 2009-2014. Madrid: Unidad de Mujeres y Ciencia de la Secretaría de Estado de Investigación, Desarrollo e Innovación del Ministerio de Economía y Competitividad.

ELIZONDO, ARANTXA, NOVO, AINHOA \& SILVESTRE, MARÍA (2010): Igualdad de mujeres y hombres en las universidades españolas. Madrid: Instituto de la Mujer.

EUROPEAN COMMISSION (2013). She Figures 2012: Women and Science, Statistics and Indicators. Luxembourg: Publications Office of the European Union

GALA, CAROLINA (2010): Llei, dones i universitat: vells i nous reptes. In: Doctes, doctores i catedràtiques: Cent anys d'accés lliure de la dona a la Universitat. Barcelona: Consell Interuniversitari de Catalunya

GARCÍA PRINCE, EVANGELINA (2006): El espejismo de la igualdad: el peso de las mujeres y de lo femenino en las iniciativas de cambio institucional. Otras Miradas, 6(1), 24-30.

HARDING, SANDRA (1996): Ciencia y feminismo. Madrid: Ediciones Morata.

INSTITUTO EUROPEO DE LA IGUALDAD DE GÉNERO (2012): Informe Anual 2011. Luxemburgo: Oficina de Publicaciones de la Unión Europea

LAGARDE, MARCELA (1993): Los cautiverios de las mujeres. Madresposas, monjas, putas, presas y locas. UNAM, México

LEÓN, CONSUELO (2010): La dona treballadora i la nova realitat laboral. El repte d'harmonitzar treball, família i vida personal avui. In: Doctes, doctores $i$ 
catedràtiques: Cent anys d'accés lliure de la dona a la Universitat. Barcelona: Consell Interuniversitari de Catalunya

ALBERT LÓPEZ-IBOR, ROCÍO; ESCOT MANGAS, LORENZO \& FERNÁNDEZ CORNEJO, JOSÉ ANDRÉS (2010): La predisposición de las estudiantes universitarias a auto-limitarse profesionalmente en el futuro por razones de conciliación. Estudios de economía aplicada, 28(1), 1-32

MARUANI, MARGARET; ROGERAT, CHANTAL \& TORNS, TERESA (2000): Las nuevas fronteras de la desigualdad. Hombres y mujeres en el mercado de trabajo. Barcelona: Icaria.

MASSACHUSETTS INSTITUTE OF TECHNOLOGY (1999): A Study on the Status of Women Faculty in Science at MIT. The MIT Faculty Newsletter Special Edition XI, 4.

MINISTERIO DE EDUCACIÓN, CULTURA Y DEPORTE (2016): Las cifras de la educación en España. Curso 2015-2016. Madrid: Ministerio de Educación.

MOSS-RACUSINA, CORINNE A; DOVIDIOB, JOHN F; BRESCOLLC, VICTORIA L; GRAHAMA, MARK J. \& HANDELSMAN, JO (2012): Science faculty's subtle gender biases favor male students. Proceedings of the National Academy of Sciences, 109 (41), pp. 16474-16479.

PASTOR, INMA; ACOSTA, ANA; BELZUNEGUI, ANGEL \& PONTÓN, PALOMA (2015): Where are the female entrepreneurs in Spain? The labour participation of women in the spanish university spin-offs. Women's Voices in Management: Identifying Innovative and Responsible Solutions. London: Pallgrave Macmillan

PASTOR, INMA \& BELZUNEGUI, ANGEL (2008): Dones $i$ homes a la URV. Tarragona: Universitat Rovira i Virgili.

PASTOR, INMA, PONTÓN, PALOMA, BELZUNEGUI, ANGEL Y ACOSTA, ANA (2015): Gender Policies in Spanish Universities: From Regulation to Equality Plans. Women's Voices in Management: Identifying Innovative and Responsible Solutions. London: Pallgrave Macmillan

PASTOR, INMA; SOLSONA, GLORIA \& VALLS, FRANCESC (2010): Desigualtat a la universitat per raó de gènere? La situació de les dones a la Universitat Rovira i Virgili: de la diagnosi a les mesures d'igualtat. Revista Catalana de Sociologia, 25, pp. 55-72

SÁNCHEZ DE MADARIAGA, INÉS. ET AL. (2014): Científicas en cifras 2013. Estadísticas e indicadores de la (des)igualdad de género en la formación y profesión científica. Madrid: Ministerio de Economía y Competitividad. 
SÁNCHEZ DE MADARIAGA, INÉS; RICA, SARA \& DOLADO, JOSÉ JUAN. (2011): Libro Blanco. Situación de las mujeres en la ciencia española. Madrid: Unidad de Mujeres y Ciencia, Ministerio de Ciencia e Innovación.

SCHIEBINGER, LONDA (2007): Getting More Women into Science: Knowledge Issues. Harvard Journal of Law \& Gender, 30, 365-378.

SCHIEBINGER, LONDA (2008): Gendered innovations in science and engineering. Stanford, Calif.: Stanford University Press.

SCHIEBINGER, LONDA Y KLINGE, INEKE (2010): Gendered innovations. Mainstreaming sex and gender analysis into basic and applied research. Metaanalysis of gender and science research.

PÉREZ SEDEÑO, EULALIA (2000): ¿El poder de una ilusión?: Ciencia, Género y Feminismo. In M. T. López de la Vieja (ed.), Feminismo: del pasado al presente. Ediciones Universidad de Salamanca, 2000

WENNERÅS, CHRISTINE Y WOLD, AGNES (1997): Nepotism and sexism in peer review. Nature, 387, 341-343.

ZINOVYEVA, NATALIA Y BAGUÉS, MANUEL (2011): Does gender matter for academic promotion? Evidence from a randomized natural experiment. IZA Discussion Paper, 5537. 